\title{
Determination of Mean Activity Coefficients of Metal Sulfates in Aqueous Sulfuric Acid-Sulfate Systems
}

\author{
Yasuhiro AwAKurA*, Yasushi TAKeshima*, Hiroshi Kondo*, and \\ Hiroshi MAJIMA*
}

\begin{abstract}
Mean activity coefficients of $\mathrm{Na}_{2} \mathrm{SO}_{4}, \mathrm{Li}_{2} \mathrm{SO}_{4}$ and $\mathrm{MgSO}_{4}$ were determined at $298 \mathrm{~K}$ by means of a graphic integration method applying the Gibbs-Dühem equation to aqueous $\mathrm{H}_{2} \mathrm{SO}_{4}-\mathrm{Na}_{2} \mathrm{SO}_{4}$, $\mathrm{H}_{2} \mathrm{SO}_{4}-\mathrm{Li}_{2} \mathrm{SO}_{4}$ and $\mathrm{H}_{2} \mathrm{SO}-\mathrm{MgSO}_{4}$ systems. The mean activity coefficients of these metal sulfates decreased with an increase in $\mathrm{H}_{2} \mathrm{SO}_{4}$ concentration. This activity coefficient decrease was most distinct at low concentrations of these metal sulfates. The effect of $\mathrm{H}_{2} \mathrm{SO}_{4}$ on the mean activity coefficients of these metal sulfates was compared. It was found that the magnitude of $r_{ \pm}$of these sulfates studied was in the order of $\mathrm{Li}_{2} \mathrm{SO}_{4}>\mathrm{Na}_{2} \mathrm{SO}_{4}>\mathrm{MgSO}_{4}$ at the same molality.
\end{abstract}

\section{Introduction}

A knowledge of the activities of electrolytes and their constituent ions is important in understanding the reaction kinetics in many hydrometallurgical processes such as the leaching of ores and the purification of leach solutions, as described in a previous paper ${ }^{11}$.

Measurements of the activities of acid and the constituent ions in aqueous $\mathrm{HCl}-\mathrm{MCl}_{n}$ and $\mathrm{H}_{2} \mathrm{SO}_{4}-\mathrm{M}_{n} \mathrm{SO}_{4}$ systems ( $\mathrm{M}$ : alkali or alkaline earth metals) have been made in authors' laboratory ${ }^{2 \sim 6)}$. If the activities of $\mathrm{H}_{2} \mathrm{O}$ and $\mathrm{H}_{2} \mathrm{SO}_{4}$ in aqueous $\mathrm{H}_{2} \mathrm{SO}_{4}-\mathrm{M}_{n} \mathrm{SO}_{4}$ system are known, the activity of $\mathrm{M}_{n} \mathrm{SO}_{4}$ can be determined by applying Gibbs-Dühem equation. In a previous study, the authors have measured the activities of $\mathrm{H}_{2} \mathrm{SO}_{4}$ in aqueous $\mathrm{H}_{2} \mathrm{SO}_{4}-\mathrm{M}_{n} \mathrm{SO}_{4}$ system by measuring the electromotive force of a cell consisting of a Pt-Pt black electrode and a $\mathrm{PbO}_{2}-$ $\mathrm{PbSO}_{4}$ electrode. The activities of $\mathrm{H}_{2} \mathrm{O}$ in the same solution system were also determined by means of an isopiestic method ${ }^{6}$.

This paper describes the activity coefficients of $\mathrm{M}_{n} \mathrm{SO}_{4}$ (including $\mathrm{Na}_{2} \mathrm{SO}_{4}, \mathrm{Li}_{2} \mathrm{SO}_{4}$ and $\mathrm{Mg}$ $\mathrm{SO}_{4}$ ) as calculated by using Gibbs-Dühem equation.

\section{Theoretical Background for the Determination of Activity Coef- ficients of Sulfates in Aqueous $\mathrm{H}_{2} \mathrm{SO}_{4}-\mathrm{M}_{n} \mathrm{SO}_{4}$ system}

\footnotetext{
* Department of Metallurgy, Kyoto University (Sakyo-ku, Kyoto 606)

Key Words: Mean Activity Coefficient, Metal Sulfate, Sulfuric Acid Solution, Gibbs-Dühem Equation
}

The theoretical background and experimental procedures for the determination of the activity of $\mathrm{H}_{2} \mathrm{SO}_{4}$ in aqueous $\mathrm{H}_{2} \mathrm{SO}_{4}-\mathrm{M}_{n} \mathrm{SO}_{4}$ system, applying electromotive force measurement techniques, were described in a previous paper". The activity of $\mathrm{H}_{2} \mathrm{O}$ in the same solution system, as determined by an isopiestic method, and the theoretical background as well as experimental procedures were reported in a separate paper ${ }^{6}$.

The activity coefficients of $\mathrm{M}_{n} \mathrm{SO}_{4}$ in aqueous $\mathrm{H}_{2} \mathrm{SO}_{4}-\mathrm{M}_{n} \mathrm{SO}_{4}$ system can be calculated by applying the Gibbs-Dühem relation, using activity data determined separately.

Denoting $\mathrm{H}_{2} \mathrm{O}, \mathrm{H}_{2} \mathrm{SO}_{4}$ and $\mathrm{M}_{n} \mathrm{SO}_{4}$ by suffixes w, 1 and 2, respectively, the Gibbs-Dühem equation for this particular solution system can be expressed as :

$$
\begin{gathered}
55.51 \mathrm{~d} \ln a_{\mathrm{w}}+m_{1} \mathrm{~d} \ln a_{1} \\
+m_{2} \mathrm{~d} \ln a_{2}=0
\end{gathered}
$$

The activity of $\mathrm{H}_{2} \mathrm{O}$ in aqueous $\mathrm{H}_{2} \mathrm{SO}_{4}-\mathrm{Na}_{2} \mathrm{SO}_{4}$ (or $\mathrm{Li}_{2} \mathrm{SO}_{4}$ ) system can be expressed as a function of the osmotic coefficient of water, $\phi$ by equation (2).

$$
55.51 \ln a_{\mathrm{w}}=-3\left(m_{1}+m_{2}\right) \phi
$$

$a_{1}$ and $a_{2}$ of the same solution system are given by equations (3) and (4), respectively.

$$
\begin{aligned}
& a_{1}=4 r_{ \pm, 1}{ }^{3} m_{1}{ }^{2}\left(m_{1}+m_{2}\right) \\
& a_{2}=4 r_{ \pm, 2}{ }^{3} m_{2}{ }^{2}\left(m_{1}+m_{2}\right)
\end{aligned}
$$

Inserting equations (2)-(4) into equation (1), we obtain equation (5).

$$
\begin{array}{r}
\left(m_{1}+m_{2}\right) \mathrm{d} \phi+(\phi-1) \mathrm{d} m_{1}+(\phi-1) \\
\mathrm{d} m_{2}-m_{1} \mathrm{~d} \ln \tau_{ \pm, 1}=m_{2} \mathrm{~d} \ln \gamma_{ \pm, 2}
\end{array}
$$

when $m_{2}=m_{2}{ }^{\circ}$ (constant), then $\mathrm{d} m_{2}=0$. Here $m_{2}^{\circ}$ denotes the molality of $\mathrm{Na}_{2} \mathrm{SO}_{4}$ (or $\mathrm{Li}_{2} \mathrm{SO}_{4}$ ) in a binary $\mathrm{Na}_{2} \mathrm{SO}_{4}$ (or $\mathrm{Li}_{2} \mathrm{SO}_{4}$ ) $-\mathrm{H}_{2} \mathrm{O}$ system. 
The integration of equation (5) at a constant $m_{2}^{\circ}$ value yields equation (6).

$2.303 m_{2}^{\circ} \log \left(\gamma_{ \pm, 2} / r_{ \pm, 2}{ }^{\circ}\right)=$

$$
\begin{gathered}
\int_{0}^{m_{1}}\left(m_{1}+m_{2}{ }^{0}\right) \mathrm{d} \phi+\int_{0}^{m_{1}}(\phi-1) \mathrm{d} m_{1} \\
-2.303 \int_{0}^{m_{1}} m_{1} \mathrm{~d} \log r_{ \pm, 1}
\end{gathered}
$$

where $\gamma_{ \pm, 2}^{\circ}$ is a mean activity coefficient of $\mathrm{Na}_{2} \mathrm{SO}_{4}$ (or $\mathrm{Li}_{2} \mathrm{SO}_{4}$ ) at its concentration of $m_{2}{ }^{\circ}$ in a binary $\mathrm{Na}_{2} \mathrm{SO}_{4}$ (or $\mathrm{Li}_{2} \mathrm{SO}_{4}$ ) $-\mathrm{H}_{2} \mathrm{O}$ system.

For aqueous $\mathrm{H}_{2} \mathrm{SO}_{4}-\mathrm{MgSO}_{4}$ system, equations (2) $-(6)$ can be replaced by equations $\left(2^{\prime}\right)-\left(6^{\prime}\right)$, respectively.

$$
\begin{aligned}
& 55.51 \ln a_{\mathrm{w}}=-\left(3 m_{1}+2 m_{2}\right) \phi \\
& a_{1}=4 \gamma_{ \pm, 1}{ }^{3} m_{1}{ }^{2}\left(m_{1}+m_{2}\right) \\
& a_{2}=r_{ \pm .2}{ }^{2} m_{2}\left(m_{1}+m_{2}\right) \\
& \left(3 m_{1}+2 m_{2}\right) \mathrm{d} \phi+3(\phi-1) \mathrm{d} m_{1} \\
& \quad+2(\phi-1) \mathrm{d} m_{2}-3 m_{1} \mathrm{~d} \ln \gamma_{ \pm, 1} \\
& \quad=2 m_{2} \mathrm{~d} \ln \gamma_{ \pm, 2} \\
& 4.606 m_{2}^{\circ} \log \left(\gamma_{ \pm, 2} / \gamma_{ \pm, 2}^{\circ}\right) \\
& \quad=\int_{0}^{m_{1}}\left(3 m_{1}+2 m_{2}\right) \mathrm{d} \phi+ \\
& 3 \int_{0}^{m_{1}}(\phi-1) \mathrm{d} m_{1}-6.909 \\
& \int_{0}^{m_{1}} m_{1} \mathrm{~d} \log \gamma_{ \pm, 1}
\end{aligned}
$$

The graphic integration of each term on the right hand side of equations (6) and $\left(6^{\prime}\right)$ from $m_{1}=0$ to $m_{1}=m_{1}$ under the condition of $m_{2}=$ $m_{2}^{\circ}$ enables us to determine the activity coefficient of metal sulfate, $\mathrm{M}_{n} \mathrm{SO}_{4}$, for aqueous $\mathrm{H}_{2} \mathrm{SO}_{4}-\mathrm{M}_{n} \mathrm{SO}_{4}$ system.

\section{Data Used for the Determination of the Mean Activity Coefficients of Metal Sulfates}

As mentioned before, the authors and their coworkers determined the activities of $\mathrm{H}_{2} \mathrm{SO}_{4}$ and $\mathrm{H}_{2} \mathrm{O}$ in aqueous $\mathrm{H}_{2} \mathrm{SO}_{4}-\mathrm{Na}_{2} \mathrm{SO}_{4}, \mathrm{H}_{2} \mathrm{SO}_{4}-$ $\mathrm{Li}_{2} \mathrm{SO}_{4}$ and $\mathrm{H}_{2} \mathrm{SO}_{4}-\mathrm{MgSO}_{4}$ systems in separate studies ${ }^{1 \sim 6)}$. However, these data cannot be used directly in the present calculation since the osmotic coefficients, $\phi$ and the mean activity coefficients of $\mathrm{H}_{2} \mathrm{SO}_{4}, r_{ \pm, 1}$, at a constant metal sulfate concentration $m_{2}=m_{2}^{\circ}$ are required for this calculation. In this study, the necessary values of $\phi$ and $\gamma_{ \pm, 1}$ were determined by means of both interpolation and extrapolation based on the activity values determined in our laboratory and on the thermodynamic data previously published ${ }^{7}$.

Tables I to III show the values of $\phi$ and $\tau_{ \pm, 1}$ thus determined at $m_{2}=m_{2}{ }^{\circ}$. These values are used for the calculation of $\gamma_{ \pm, 2}$ in aqueous $\mathrm{H}_{2} \mathrm{SO}_{4}-\mathrm{M}_{n} \mathrm{SO}_{4}$ system.

\section{Results and Discussion}

A typical example of graphic integration is shown in Fig. 1. In this particular example, thermodynamic data for aqueous $\mathrm{H}_{2} \mathrm{SO}_{4}-\mathrm{Li}_{2} \mathrm{SO}_{4}$ system at $298 \mathrm{~K}$ were used. Figures 1 (a), 1(b) and 1 (c) correspond to the first term, second term and third term, respectively, on the right hand side of equation (6). By integrating graphically from $m_{1}=0$ to $m_{1}=m_{1}$, we can determine $\gamma_{ \pm, 2}$ at a constant level of $m_{2}=m_{2}{ }^{\circ}$ from equation (6).

Similarly the values of $\gamma_{ \pm}$of $\mathrm{Na}_{2} \mathrm{SO}_{4}$ and $\mathrm{MgSO}_{4}$ in aqueous $\mathrm{H}_{2} \mathrm{SO}_{4}-\mathrm{Na}_{2} \mathrm{SO}_{4}$ and $\mathrm{H}_{2} \mathrm{SO}_{4}-$ $\mathrm{MgSO}_{4}$ systems, respectively, can be determined by graphic integration.

The values of $\gamma_{ \pm, 2}$ for aqueous $\mathrm{H}_{2} \mathrm{SO}_{4}-\mathrm{Na}_{2}$ $\mathrm{SO}_{4}, \mathrm{H}_{2} \mathrm{SO}_{4}-\mathrm{Li}_{2} \mathrm{SO}_{4}$ and $\mathrm{H}_{2} \mathrm{SO}_{4}-\mathrm{MgSO}_{4}$ systems calculated in this manner at $298 \mathrm{~K}$ are listed in the last column of Tables I to III.
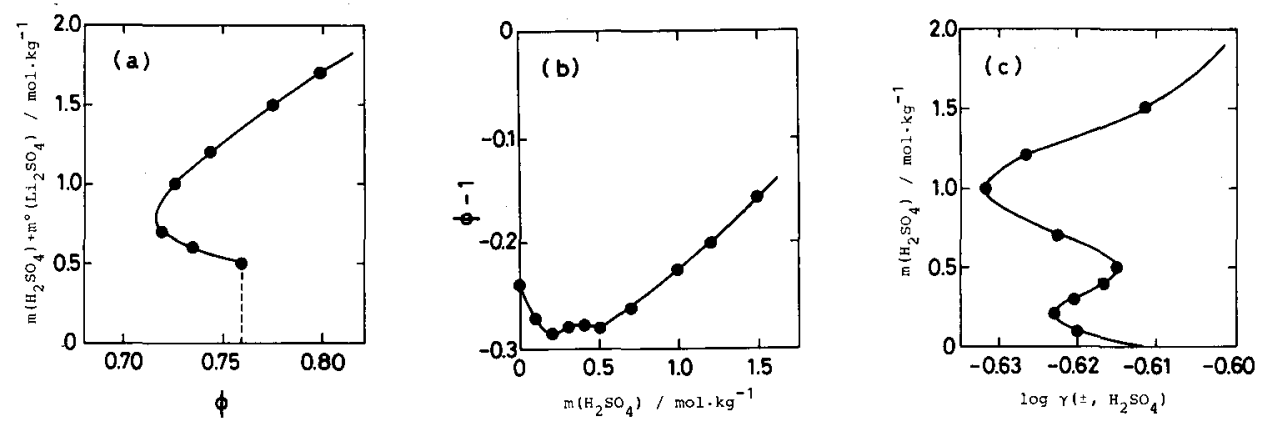

Fig. 1 A typical example of graphic integration at $m\left(\mathrm{Li}_{2} \mathrm{SO}_{4}\right)=0.5 \mathrm{~mol} \cdot \mathrm{kg}^{-1}$ (Example employed : aqueous $\mathrm{H}_{2} \mathrm{SO}_{4}-\mathrm{Li}_{2} \mathrm{SO}_{4}$ system) 
Table 1 Thermodynamic data used for the calculation of mean activity coefficients of $\mathrm{Na}_{2} \mathrm{SO}_{4}$ in aqueous $\mathrm{H}_{2} \mathrm{SO}_{4}-\mathrm{Na}_{2} \mathrm{SO}_{4}$ system at $298 \mathrm{~K}$

\begin{tabular}{|c|c|c|c|c|c|}
\hline$\frac{m\left(\mathrm{Na}_{2} \mathrm{SO}_{4}\right)}{\mathrm{mol} \cdot \mathrm{kg}^{-1}}$ & $\frac{m\left(\mathrm{H}_{2} \mathrm{SO}_{4}\right)}{\mathrm{mol} \cdot \mathrm{kg}^{-1}}$ & $a\left(\mathrm{H}_{2} \mathrm{O}\right)$ & $\phi$ & $\begin{array}{l}\log \gamma( \pm \\
\left.\mathrm{H}_{2} \mathrm{SO}_{4}\right)\end{array}$ & $\begin{array}{l}\log \gamma( \pm \\
\left.\mathrm{Na}_{2} \mathrm{SO}_{4}\right)\end{array}$ \\
\hline \multirow{14}{*}{0.1} & 0 & & $0.787^{*}$ & $-0.556^{* *}$ & $-0.344^{* * *}$ \\
\hline & 0.1 & 0.9929 & 0.659 & -0.688 & -0.462 \\
\hline & 0.2 & 0.9893 & 0.664 & -0.769 & -0.491 \\
\hline & 0.3 & 0.9860 & 0.652 & -0.800 & -0.581 \\
\hline & 0.4 & 0.9822 & 0.665 & -0.824 & -0.625 \\
\hline & 0.5 & 0.9788 & 0.661 & -0.842 & -0.699 \\
\hline & 0.6 & 0.9750 & 0.669 & -0.860 & -0.719 \\
\hline & 0.7 & 0.9711 & 0.678 & -0.876 & -0.726 \\
\hline & 0.8 & 0.9672 & 0.686 & -0.889 & -0.738 \\
\hline & 0.9 & 0.9630 & 0.698 & -0.899 & -0.733 \\
\hline & 1.0 & 0.9588 & 0.708 & -0.907 & -0.750 \\
\hline & 1.1 & 0.9543 & 0.721 & -0.912 & -0.750 \\
\hline & 1.2 & 0.9497 & 0.735 & -0.914 & -0.777 \\
\hline & 1.3 & 0.9451 & 0.746 & -0.914 & -0.818 \\
\hline \multirow{14}{*}{0.2} & 0 & & $0.748^{*}$ & $-0.717^{* *}$ & $-0.431 * * *$ \\
\hline & 0.1 & 0.9895 & 0.651 & -0.792 & -0.533 \\
\hline & 0.2 & 0.9859 & 0.657 & -0.832 & -0.573 \\
\hline & 0.3 & 0.9827 & 0.646 & -0.851 & -0.638 . \\
\hline & 0.4 & 0.9791 & 0.651 & -0.864 & -0.684 \\
\hline & 0.5 & 0.9756 & 0.653 & -0.876 & -0.733 \\
\hline & 0.6 & 0.9718 & 0.662 & -0.888 & -0.759 \\
\hline & 0.7 & 0.9679 & 0.671 & -0.899 & -0.783 \\
\hline & 0.8 & 0.9640 & 0.678 & -0.908 & -0.801 \\
\hline & 0.9 & 0.9598 & 0.690 & -0.915 & -0.812 \\
\hline & 1.0 & 0.9556 & 0.700 & -0.920 & -0.830 \\
\hline & 1.1 & 0.9512 & 0.712 & -0.925 & -0.824 \\
\hline & 1.2 & 0.9465 & 0.727 & -0.928 & -0.824 \\
\hline & 1.3 & 0.9423 & 0.733 & -0.929 & -0.857 \\
\hline \multirow{12}{*}{0.5} & 0 & & $0.693^{*}$ & $-1.053^{* *}$ & $-0.569 * * *$ \\
\hline & 0.1 & 0.9792 & 0.648 & -0.986 & -0.625 \\
\hline & 0.2 & 0.9760 & 0.642 & -0.965 & -0.666 \\
\hline & 0.3 & 0.9731 & 0.631 & -0.961 & -0.706 \\
\hline & 0.4 & 0.9697 & 0.633 & -0.958 & -0.740 \\
\hline & 0.5 & 0.9661 & 0.638 & -0.957 & -0.767 \\
\hline & 0.6 & 0.9623 & 0.646 & -0.957 & -0.790 \\
\hline & 0.7 & 0.9583 & 0.657 & -0.957 & -0.810 \\
\hline & 0.8 & 0.9545 & 0.663 & -0.957 & -0.833 \\
\hline & 0.9 & 0.9501 & 0.677 & -0.957 & -0.845 \\
\hline & 1.0 & 0.9458 & 0.687 & -0.957 & -0.860 \\
\hline & 1.1 & 0.9414 & 0.698 & -0.956 & -0.873 \\
\hline \multirow{9}{*}{1.0} & 0 & & $0.644^{*}$ & $-1.218^{* *}$ & $-0.690 * * *$ \\
\hline & 0.1 & 0.9630 & 0.634 & -1.147 & -0.714 \\
\hline & 0.2 & 0.9600 & 0.630 & -1.098 & -0.740 \\
\hline & 0.3 & 0.9570 & 0.626 & -1.079 & -0.762 \\
\hline & 0.4 & 0.9538 & 0.625 & -1.069 & -0.783 \\
\hline & 0.5 & 0.9503 & 0.629 & -1.060 & -0.799 \\
\hline & 0.6 & 0.9466 & 0.635 & -1.048 & -0.818 \\
\hline & 0.7 & 0.9429 & 0.640 & -1.033 & -0.839 \\
\hline & 0.8 & 0.9385 & 0.653 & -1.020 & -0.854 \\
\hline
\end{tabular}

\begin{tabular}{|c|c|c|c|c|c|}
\hline$\frac{m\left(\mathrm{Li}_{2} \mathrm{SO}_{4}\right)}{\mathrm{mol} \cdot \mathrm{kg}^{-1}}$ & $\frac{\frac{m\left(\mathrm{H}_{2} \mathrm{SO}_{4}\right)}{\mathrm{mol} \cdot \mathrm{kg}^{-1}}}{\mid}$ & $a\left(\mathrm{H}_{2} \mathrm{O}\right)$ & $\phi$ & $\begin{array}{l}\log \gamma( \pm, \\
\left.\mathrm{H}_{2} \mathrm{SO}_{4}\right)\end{array}$ & $\begin{array}{l}\log \gamma( \pm \\
\left.\mathrm{Li}_{2} \mathrm{SO}_{4}\right)\end{array}$ \\
\hline \multirow{6}{*}{1.5} & 0 & & $0.621^{*}$ & $-1.253^{* *}$ & $-0.764^{* * *}$ \\
\hline & 0.1 & 0.9470 & 0.630 & -1.200 & -0.772 \\
\hline & 0.2 & 0.9443 & 0.624 & -1.168 & -0.790 \\
\hline & 0.3 & 0.9413 & 0.622 & -1.152 & -0.804 \\
\hline & 0.4 & 0.9381 & 0.622 & -1.142 & -0.818 \\
\hline & 0.5 & 0.9346 & 0.626 & -1.135 & -0.830 \\
\hline \multicolumn{6}{|c|}{$\begin{array}{l}\text { * calculated from mean activity coefficients of } \mathrm{Na}_{2} \mathrm{SO}_{4} \text { in } \\
\mathrm{Na}_{2} \mathrm{SO}_{4}-\mathrm{H}_{2} \mathrm{O} \text { binary system by using Gibbs-Dühem } \\
\text { equation. }\end{array}$} \\
\hline
\end{tabular}

Figure 2 shows the effect of $\mathrm{H}_{2} \mathrm{SO}_{4}$ concentration on $r_{ \pm}$of $\mathrm{Na}_{2} \mathrm{SO}_{4}$ at a constant $\mathrm{Na}_{2} \mathrm{SO}_{4}$ concentration. As may be seen in this figure, $\gamma_{ \pm}$of $\mathrm{Na}_{2} \mathrm{SO}_{4}$ at a low $\mathrm{Na}_{2} \mathrm{SO}_{4}$ level, such as $0.1 \mathrm{~mol} \cdot \mathrm{kg}^{-1}$, decreases sharply with an increase in $\mathrm{H}_{2} \mathrm{SO}_{4}$ concentration. On the contrary, the effect of $\mathrm{H}_{2} \mathrm{SO}_{4}$ concentration on $\gamma_{ \pm}$of $\mathrm{Na}_{2} \mathrm{SO}_{4}$ in 1.0 and $1.5 \mathrm{~mol} \cdot \mathrm{kg}^{-1} \mathrm{Na}_{2} \mathrm{SO}_{4}$ solutions was less than that in dilute solutions. The values of $\gamma_{ \pm}$of $\mathrm{Na}_{2} \mathrm{SO}_{4}$ in concentrated $\mathrm{Na}_{2} \mathrm{SO}_{4}$ solutions also decrease with an increase in $\mathrm{H}_{2} \mathrm{SO}_{4}$ concentration.

Figure 3 depicts the effect of $\mathrm{H}_{2} \mathrm{SO}_{4}$ concen-

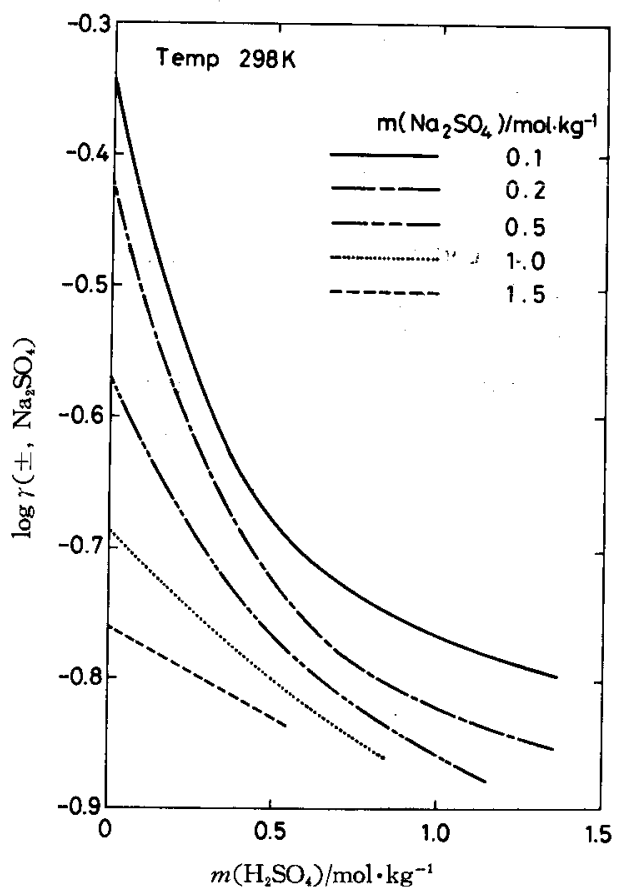

Fig. 2 Mean activity coefficients of $\mathrm{Na}_{2} \mathrm{SO}_{4}$ in aqueous $\mathrm{H}_{2} \mathrm{SO}_{4}-\mathrm{Na}_{2} \mathrm{SO}_{4}$ system at $298 \mathrm{~K}$ 
Table 2 Thermodynamic data used for the calculation of mean activity coefficients of $\mathrm{Li}_{2} \mathrm{SO}_{4}$ in aqueous $\mathrm{H}_{2} \mathrm{SO}_{4}-\mathrm{Li}_{2} \mathrm{SO}_{4}$ system at $298 \mathrm{~K}$

\begin{tabular}{|c|c|c|c|c|c|}
\hline$\frac{m\left(\mathrm{Li}_{2} \mathrm{SO}_{4}\right)}{\mathrm{mol} \cdot \mathrm{kg}^{-1}}$ & $\frac{m\left(\mathrm{H}_{2} \mathrm{SO}_{4}\right)}{\mathrm{mol} \cdot \mathrm{kg}^{-1}}$ & $a\left(\mathrm{H}_{2} \mathrm{O}\right)$ & $\phi$ & $\begin{array}{l}\log \gamma( \pm \\
\left.\mathrm{H}_{2} \mathrm{SO}_{4}\right)\end{array}$ & $\begin{array}{l}\log \gamma( \pm \\
\left.\mathrm{Li}_{2} \mathrm{SO}_{4}\right)\end{array}$ \\
\hline \multirow{7}{*}{0.1} & 0 & & $0.798^{*}$ & $-0.671 * *$ & $-0.321 * * *$ \\
\hline & 0.1 & 0.9923 & 0.715 & -0.723 & -0.460 \\
\hline & 0.2 & 0.9889 & 0.689 & -0.766 & -0.553 \\
\hline & 0.4 & 0.9814 & 0.695 & -0.806 & -0.708 \\
\hline & 0.5 & 0.9779 & 0.689 & -0.826 & -0.735 \\
\hline & 0.7 & 0.9703 & 0.697 & -0.863 & -0.759 \\
\hline & 1.5 & 0.9338 & 0.792 & -0.897 & -0.900 \\
\hline \multirow{9}{*}{0.2} & 0 & & $0.778^{*}$ & $-0.736 * *$ & $-0.391 * * *$ \\
\hline & 0.1 & 0.9883 & 0.726 & -0.794 & -0.462 \\
\hline & 0.2 & 0.9850 & 0.699 & -0.817 & -0.529 \\
\hline & 0.3 & 0.9816 & 0.687 & -0.828 & -0.592 \\
\hline & 0.4 & 0.9779 & 0.689 & -0.840 & -0.636 \\
\hline & 0.5 & 0.9740 & 0.696 & -0.852 & -0.674 \\
\hline & 0.7 & 0.9661 & 0.709 & -0.880 & -0.693 \\
\hline & 1.2 & 0.9435 & 0.769 & -0.906 & -0.728 \\
\hline & 1.5 & 0.9287 & 0.805 & -0.904 & -0.757 \\
\hline \multirow{10}{*}{0.5} & 0 & & $0.761^{*}$ & $-0.913^{* *}$ & $-0.487^{* * *}$ \\
\hline & 0.1 & 0.9764 & 0.737 & -0.920 & -0.520 \\
\hline & 0.2 & 0.9733 & 0.715 & -0.923 & -0.554 \\
\hline & 0.3 & 0.9691 & 0.726 & -0.922 & -0.580 \\
\hline & 0.4 & 0.9654 & 0.724 & -0.919 & -0.602 \\
\hline & 0.5 & 0.9616 & 0.725 & -0.916 & -0.623 \\
\hline & 0.7 & 0.9530 & 0.742 & -0.921 & -0.648 \\
\hline & 1.0 & 0.9390 & 0.776 & -0.932 & -0.652 \\
\hline & 1.2 & 0.9293 & 0.798 & -0.926 & -0.670 \\
\hline & 1.5 & 0.9137 & 0.835 & -0.913 & -0.701 \\
\hline \multirow{9}{*}{1.0} & 0 & & $0.783^{*}$ & $-1.023^{* *}$ & $-0.548^{* * *}$ \\
\hline & 0.1 & 0.9546 & 0.782 & -1.013 & -0.559 \\
\hline & 0.2 & 0.9510 & 0.775 & -1.006 & -0.572 \\
\hline & 0.3 & 0.9470 & 0.775 & -0.999 & -0.585 \\
\hline & 0.4 & 0.9428 & 0.779 & -0.992 & -0.597 \\
\hline & 0.5 & 0.9389 & 0.778 & -0.985 & -0.607 \\
\hline & 0.7 & 0.9301 & 0.789 & -0.974 & -0.611 \\
\hline & 1.0 & 0.9159 & 0.813 & -0.962 & -0.642 \\
\hline & 1.2 & 0.9059 & 0.831 & -0.949 & -0.654 \\
\hline \multirow{6}{*}{1.5} & 0 & & $0.816^{*}$ & $-1.073^{* *}$ & $-0.565^{* * *}$ \\
\hline & 0.1 & 0.9323 & 0.811 & -1.059 & -0.573 \\
\hline & 0.2 & 0.9286 & 0,806 & -1.047 & -0.582 \\
\hline & 0.3 & 0.9246 & 0.806 & -1.040 & -0.590 \\
\hline & 0.4 & 0.9206 & 0.806 & -1.034 & -0.597 \\
\hline & 0.5 & 0.9163 & 0.809 & -1.026 & -0.604 \\
\hline
\end{tabular}

* calculated from mean activity coefficients of $\mathrm{Li}_{2} \mathrm{SO}_{4}$ in $\mathrm{Li}_{2} \mathrm{SO}_{4}-\mathrm{H}_{2} \mathrm{O}$ binary system by using Gibbs-Dühem equation.

** extrapolated from mean activity coefficients of $\mathrm{H}_{2} \mathrm{SO}_{4}$ as determined in the present study.

*** after Dobos ${ }^{7}$.
Table 3 Thermodynamic data used for the calculation of mean activitiy coefficients of $\mathrm{MgSO}_{4}$ in aqueous $\mathrm{H}_{2} \mathrm{SO}_{4}-\mathrm{MgSO}_{4}$ system at $298 \mathrm{~K}$

\begin{tabular}{|c|c|c|c|c|c|}
\hline$\frac{m\left(\mathrm{MgSO}_{4}\right)}{\mathrm{mol} \cdot \mathrm{kg}^{-1}}$ & $\frac{m\left(\mathrm{H}_{2} \mathrm{SO}_{4}\right)}{\mathrm{mol} \cdot \mathrm{kg}^{-1}}$ & $a\left(\mathrm{H}_{2} \mathrm{O}\right)$ & $\phi$ & $\begin{array}{l}\log \gamma( \pm \\
\left.\mathrm{H}_{2} \mathrm{SO}_{4}\right)\end{array}$ & $\begin{array}{l}\log r( \pm \\
\left.\mathrm{MgSO}_{4}\right)\end{array}$ \\
\hline \multirow{10}{*}{0.1} & 0 & & $0.565^{*}$ & $-0.648^{* *}$ & $-0.745^{* * *}$ \\
\hline & 0.1 & 0.9943 & 0.631 & -0.707 & -0.922 \\
\hline & 0.2 & 0.9907 & 0.645 & -0.767 & -0.991 \\
\hline & 0.4 & 0.9836 & 0.655 & -0.818 & -1.201 \\
\hline & 0.6 & 0.9763 & 0.666 & -0.850 & -1.362 \\
\hline & 0.8 & 0.9686 & 0.681 & -0.873 & -1.479 \\
\hline & 1.0 & 0.9605 & 0.699 & -0.887 & -1.595 \\
\hline & 1.2 & 0.9518 & 0.722 & -0.893 & -1.728 \\
\hline & 1.4 & 0.9425 & 0.747 & -0.895 & -1.799 \\
\hline & 1.5 & 0.9376 & 0.761 & -0.897 & -1.793 \\
\hline \multirow{10}{*}{0.2} & 0 & & $0.501^{*}$ & $-0.764^{* *}$ & $-0.959^{* * *}$ \\
\hline & 0.1 & 0.9926 & 0.586 & -0.790 & -1.049 \\
\hline & 0.2 & 0.9890 & 0.613 & -0.812 & -1.133 \\
\hline & 0.4 & 0.9818 & 0.639 & -0.845 & -1.269 \\
\hline & 0.6 & 0.9743 & 0.658 & -0.870 & -1.362 \\
\hline & 0.8 & 0.9664 & 0.677 & -0.885 & -1.444 \\
\hline & 1.0 & 0.9583 & 0.696 & -0.893 & -1.521 \\
\hline & 1.2 & 0.9492 & 0.723 & -0.896 & -1.587 \\
\hline & 1.4 & 0.9395 & 0.753 & -0.898 & -1.606 \\
\hline & 1.5 & 0.9342 & 0.771 & -0.999 & -1.585 \\
\hline \multirow{9}{*}{0.5} & 0 & & $0.484^{*}$ & $-0.925^{* *}$ & $-1.167^{* * *}$ \\
\hline & 0.2 & 0.9838 & 0.567 & -0.914 & -1.246 \\
\hline & 0.4 & 0.9760 & 0.613 & -0.910 & -1.321 \\
\hline & 0.6 & 0.9677 & 0.650 & -0.909 & -1.379 \\
\hline & 0.8 & 0.9589 & 0.686 & -0.912 & -1.412 \\
\hline & 1.0 & 0.9490 & 0.726 & -0.915 & -1.408 \\
\hline & 1.2 & 0.9399 & 0.748 & -0.912 & -1.445 \\
\hline & 1.4 & 0.9305 & 0.769 & -0.906 & -1.491 \\
\hline & 1.5 & 0.9237 & 0.801 & -0.903 & -1.449 \\
\hline \multirow{7}{*}{1.0} & 0 & & $0.500^{*}$ & $-1.017 * *$ & $-1.310^{* * *}$ \\
\hline & 0.2 & 0.9732 & 0.581 & -1.000 & -1.331 \\
\hline & 0.4 & 0.9642 & 0.633 & -0.965 & -1.367 \\
\hline & 0.6 & 0.9547 & 0.677 & -0.944 & -1.393 \\
\hline & 0.8 & 0.9445 & 0.721 & -0.935 & -1.402 \\
\hline & 1.0 & 0.9334 & 0.765 & -0.924 & -1.406 \\
\hline & 1.2 & 0.9216 & 0.809 & -0.910 & -1.405 \\
\hline \multirow{6}{*}{1.5} & 0 & & $0.560^{*}$ & $-1.052 * *$ & $-1.372^{* * *}$ \\
\hline & 0.2 & 0.9592 & 0.642 & -1.032 & -1.368 \\
\hline & 0.4 & 0.9485 & 0.699 & -0.989 & -1.381 \\
\hline & 0.6 & 0.9374 & 0.747 & -0.959 & -1.366 \\
\hline & 0.8 & 0.9259 & 0.791 & -0.938 & -1.388 \\
\hline & 1.0 & 0.9143 & 0.829 & -0.919 & -1.381 \\
\hline
\end{tabular}

* calculated from mean activity coefficients of $\mathrm{MgSO}_{4}$ in $\mathrm{MgSO}_{4}-\mathrm{H}_{2} \mathrm{O}$ binary system by using GibbsDühem equation.

** extrapolated from mean activity coefficients of $\mathrm{H}_{2} \mathrm{SO}_{4}$ as determined in the present study.

*** after Dobos ${ }^{7}$. 


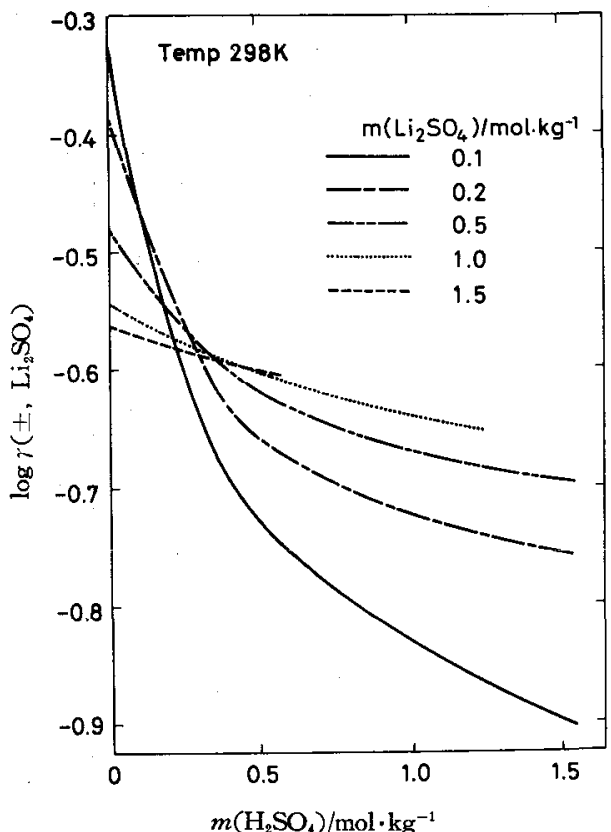

Fig. 3 Mean activity coefficients of $\mathrm{Li}_{2} \mathrm{SO}_{4}$ in aqueous $\mathrm{H}_{2} \mathrm{SO}_{4}-\mathrm{Li}_{2} \mathrm{SO}_{4}$ system at $298 \mathrm{~K}$

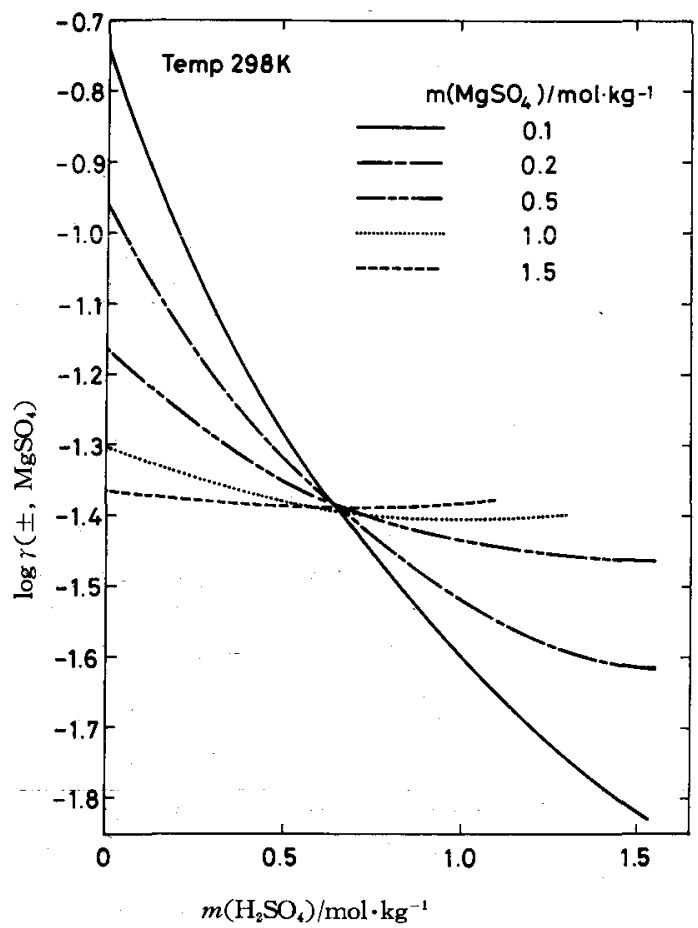

Fig. 4 Mean activity coefficients of $\mathrm{MgSO}_{4}$ in aqueous $\mathrm{H}_{2} \mathrm{SO}_{4}-\mathrm{MgSO}_{4}$ system at $298 \mathrm{~K}$

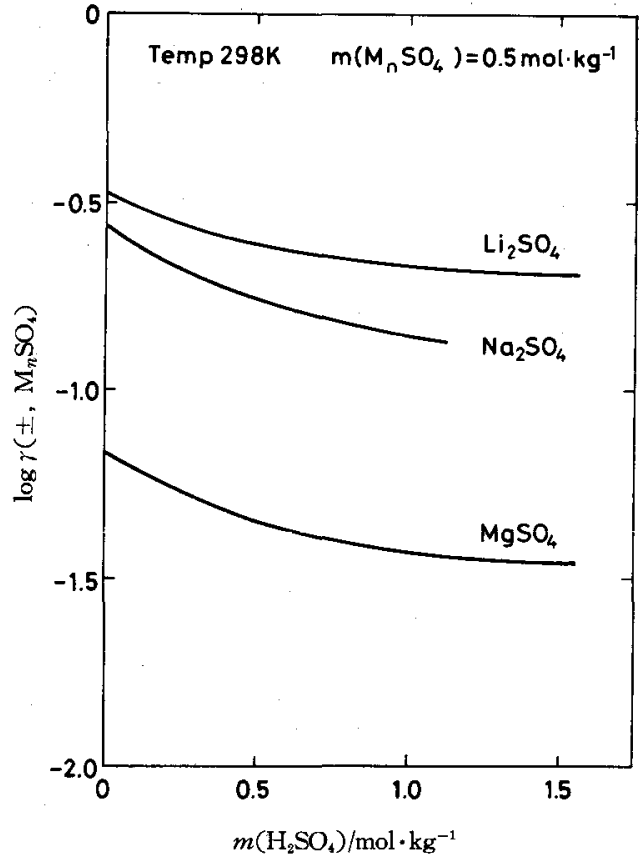

Fig. 5 A comparison of the effects of $\mathrm{H}_{2} \mathrm{SO}_{4}$ concentration on the mean activity coefficients of metal sulfates whose concentration are 0.5 $\mathrm{mol} \cdot \mathrm{kg}^{-1}$

tration on $r_{ \pm}$of $\mathrm{Li}_{2} \mathrm{SO}_{4}$ at a constant $\mathrm{Li}_{2} \mathrm{SO}_{4}$ level. Figure 4 shows a similar illustration for aqueous $\mathrm{H}_{2} \mathrm{SO}_{4}-\mathrm{MgSO}_{4}$ system. The effect of $\mathrm{H}_{2} \mathrm{SO}_{4}$ concentration on $r_{ \pm}$of $\mathrm{Li}_{2} \mathrm{SO}_{4}$ as well as on $r_{ \pm}$of $\mathrm{MgSO}_{4}$ appears similar to that on $r_{ \pm}$of $\mathrm{Na}_{2} \mathrm{SO}_{4}$.

Figure 5 shows a comparison of the effect of $\mathrm{H}_{2} \mathrm{SO}_{4}$ concentration on $\gamma_{ \pm}$of metal sulfates for aqueous $\mathrm{H}_{2} \mathrm{SO}_{4}-\mathrm{M}_{n} \mathrm{SO}_{4}$ system whose $\mathrm{M}_{n} \mathrm{SO}_{4}$ concentration is $0.5 \mathrm{~mol} \cdot \mathrm{kg}^{-1}$. The effect of $\mathrm{H}_{2} \mathrm{SO}_{4}$ on mean activity coefficients of these metal sulfates was compared. It was found that the magnitude of $\gamma_{ \pm}$of these sulfates studied was in the order of $\mathrm{Li}_{2} \mathrm{SO}_{4}>\mathrm{Na}_{2} \mathrm{SO}_{4}>\mathrm{MgSO}$, at the same molality.

\section{Conclusions}

Mean activity coefficients of $\mathrm{Na}_{2} \mathrm{SO}_{4}, \mathrm{Li}_{2} \mathrm{SO}_{4}$ and $\mathrm{MgSO}_{4}$ were determined by means of a graphic integration method applying GibbsDühem equation to aqueous $\mathrm{H}_{2} \mathrm{SO}_{4}-\mathrm{M}_{n} \mathrm{SO}_{4}$ system. For this purpose, osmotic coefficients and mean activity coefficients of $\mathrm{H}_{2} \mathrm{SO}_{4}$, determined by interpolation and extrapolation me- 
thods from the thermodynamic data of the previous works of the present authors, were used

The mean activity coefficients of these sulfates decrease with an increase in the concentration of $\mathrm{H}_{2} \mathrm{SO}_{4}$. The decreasing tendency was most distinct at low concentrations of these metal sulfates. The effect of $\mathrm{H}_{2} \mathrm{SO}_{4}$ concentration on the mean activity coefficients of these sulfates was compared. It was found that the magnitude of $\gamma_{ \pm}$of these sulfates studied was in the order of $\mathrm{Li}_{2} \mathrm{SO}_{4}>\mathrm{Na}_{2} \mathrm{SO}_{4}>\mathrm{MaSO}_{4}$ at the same molality.

\section{Acknowledgments :}

Financial support by the Ministry of Education of Japan (Grant-in-Aid for Scientific Research No.
56470051) is gratefully acknowledged.

References :

1) H. Majima and Y. Awakura, Met. Trans. B. 12 B, 141 (1981)

2) H. Majima, Y. Awakura and T. Sato, Denki Kagaku 48, 597 (1980).

3) H. Majima, Y. Awakura, T. Sato and T. Michimoto, Denki Kagaku 50, 934 (1982).

4) Y. Awakura, T. Michimoto, Y. Takeshima and H. Majima, Denki Kagaku 51, 302 (1983).

5) Y. Takeshima, H. Kondo, Y. Awakura and H. Majima, Preceedings of Spring Meeting of MMIJ, Tokyo, 1983, p. 143.

6) T. Michimoto, Y. Awakura and H. Majima, Denki Kagaku 51, 373 (1983).

7) D. Dobos, "Electrochemical Data", Elsevier Scientific Publ. Co., Amsterdam, 1975.

(Received Jun. 6, 1983; Accepted Nov. 1, 1983)

\section{$\Leftrightarrow$ 協 会}

\section{第 6 回溶液化学シンポジウム}

第6 回溶液化学シンポジウムは大阪市立大学理学部村 上幸夫教授の肝煎で, 昭和 58 年 12 月 12 日，13 日の 両日にわたり，近鉄上本町駅近くのなにわ会館で開催さ れた。春や秋に殺到する他の討論会との重複を避けるた めに，このシンポジウムは 12 月頃に開かれることが多 h.

これまでの溶液化学シンポジウムの短い歴史をふりか えってみると表1のようになる。

こらしてみると，はじめの 2〜3 年は 20〜25 件程度 だった発表論文数が，その後約 30 数件に增え，さらに 今年になって飛矅的に増加したことが示される．参加者 もこれまでは拈よそ70 80 人ぐらいであったが，今回 は 170 人にも及ぶものとなり，もはや従来の 1 会場形式 では納まらず，遂にやむを得ず 2 会場に拡張せざるを得 なくなった。

今年は 101 才の長い生涯の最後むで溶液に関する研究 に捧げられた Hildebrand 教授の逝去を悼んで特別セッ ションが設けられ，この中では特別講演 2 件を含む 6 件 の論文が発表された。また折加ら来日中の韓国高等科学 研究所の全武植 (Mu Shik Jhon) 博士が特別講演を行 うなど，国際色む盛り込んだ多彩な企画がたてられ，最 終日の最後の講演まで多数の参加者の足をひき止め, 活 発な討論が行われた。

溶液化学シンポジウムの特徵はまず発表論文の内容が
表 1

\begin{tabular}{|c|c|c|c|c|c|}
\hline & 期 & 日 & 爷 場 & $\begin{array}{l}\text { 登表論 } \\
\text { 文 数 }\end{array}$ & 備 \\
\hline 第 1 回 & 53年 4 月 & $5 \sim 6$ 日 & $\begin{array}{l}\text { 菓高太 } \\
\text { (長津田) }\end{array}$ & 22 & $\begin{array}{l}\text { 電気化学協会 } \\
\text { 第45回大会と } \\
\text { 併催 }\end{array}$ \\
\hline 第2国 & 54 年 12 月 & 12 13日 & $\begin{array}{l}\text { 東工大 } \\
\text { (無津由) }\end{array}$ & 25 & $\begin{array}{l}\text { 昭和 } 54 \text { 年度女 } \\
\text { 部省科学研究 } \\
\text { 費総合研究B } \\
\text { 乏森方ム } \\
\text { と併倠 }\end{array}$ \\
\hline 第 3 回 & 55 年10 月 & $1 \sim 2$ 日 & 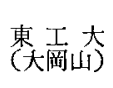 & 21 & $\begin{array}{l}\text { 雷気化学協会 } \\
\text { 愁季大会上併 } \\
\text { 雔 }\end{array}$ \\
\hline 第 4 回 & 56年11月 & $11 \sim 13$ 日 & $\begin{array}{l}\text { 静 岡 大 } \\
\text { (浜松) }\end{array}$ & 39 & $\begin{array}{l}\text { 日本熱測定学 } \\
\text { 会と共倠 }\end{array}$ \\
\hline 第 5 回 & 57年12月 & $8 \sim 10$ 日 & $\begin{array}{c}\text { 京大会館 } \\
\text { (京都) }\end{array}$ & 32 & 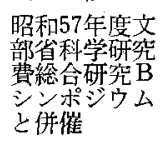 \\
\hline 第 6 回 & 58年12月 & $12 \sim 13$ 日 & $\begin{array}{l}\text { なにわ会 } \\
\text { 館(大阪) }\end{array}$ & 74 & \\
\hline
\end{tabular}

実に多種多㥞で，さまざまな理論や測定法が適用されて いる点であるう。「溶液」というきわめて広範囲で漠然 としている系を多方面からアプローチしよらとする試み がなされ，ほとんどすべての研究発表に対して熱心な質 疑応答が行われる。こういった等気が長く継続するシン ポジムであって欲しいものである。

来年は東京地区で第 7 回の溶液化学シンポジウムが開 催される予定である。

(東京工業大学総合理工学研究科 大瀧仁志) 